\title{
El debate sobre la feminización del lenguaje en la prensa francesa: análisis de chroniques de langage sobre el français inclusif en Le Figaro
}

\author{
The debate on the feminization of language and its \\ coverage in the French media: an analysis of inclusive \\ French in Le Figaro's Chroniques de langage
}

\author{
AurÉLIE Fontanille \\ Universidad de Murcia \\ aurelie.fontanille@um.es
}

\begin{abstract}
This work focuses on the debate related to the feminisation of language and, more particularly, to the inclusive French generated by the views expressed by specialists in the $L e F i$ garo newspaper at the Chronique de langage $(C D L)$ L'actu des mots. Its main aim consists of: (1) present, contextualise and justify the treatment of this social and linguistic phenomenon in a particular journalistic gender such as the columns on language (CSL) published in the digital edition of that newspaper; and (2) observe the feed-back that this type of multidirectional communication produces on the basis of the analysis of the comments issued both internally on the CSL and on social media, more specifically on Twitter. We are interested in investigating, on the one hand, this topical linguistic issue which links the notion of gender with the position of women in a changing patriarchal society and, on the other hand, the nature of these language-centred media spaces, in order to hightlight their normative orientation and ideological charge, reinforced by the web's own tools.
\end{abstract}

\begin{abstract}
Résumé
Ce travail porte sur le débat concernant la féminisation du langage et, plus particulièrement, le français inclusif généré par les opinions versées par les spécialistes du journal Le Figaro dans la chronique de langage (CDL) L'Actu des mots. Son objectif principal consiste à: 1) présenter, contextualiser et justifier le traitement donné à ce phénomène social et linguistique dans un genre journalistique singulier tel que le sont les CDL publiées dans l'édition numérique du dit journal, et 2) constater le feed-back que ce genre de communication multidirectionnelle produit à partir de l'analyse des commentaires émis aussi bien en tant que réponse interne à la CDL que sur les réseaux sociaux et plus précisément, sur Twitter. En effet, notre intérêt est axé d'un côté sur cette question linguistique d'actualité qui met en relation la notion de genre avec la position de la femme dans une société patriarcale en constante évolution et, d'un autre côté, sur la nature de ces espaces médiatiques consacrés à la langue, afin de mettre en évidence leur orientation normative et leur charge idéologique, renforcées par les propres outils qu'offrent Internet.
\end{abstract}




\section{Key-words}

columns on language (Chroniques de langage), social networks, inclusive French, ideologies, linguistic sexism.

\section{Mots clés}

Columna sobre la lengua (Chroniques de langage), réseaux sociaux, français inclusif, idéologies, sexisme linguistique.

\section{Introducción}

Este artículo tiene como propósito analizar el tratamiento de la feminización del lenguaje en las columnas sobre la lengua (CSL) de la versión digital del periódico francés más antiguo lefigaro.fr mediante las opiniones y los argumentos defendidos por especialistas, así como constatar su repercusión en el lector por medio de redes sociales en la era digital. Este fenómeno común a varios países occidentales presenta un conjunto de variaciones usadas por una comunidad epistémica totalmente consciente del papel de la lengua como transmisora de saberes.

La feminización del lenguaje o français inclusif se encuentra actualmente en el corazón de un intenso debate mediático lingüístico y sociocultural que se puede apreciar a través del corpus seleccionado formado por 23 textos periodísticos metalingüísticos sobre los procedimientos lingüísticos que este fenómeno supone y que vienen acompañados de contenidos transversales tales como la historia, el feminismo, la política y el sexismo lingüístico. Las CSL orientan a la comunidad hablante sobre los usos variados y cambiantes de la sociedad actual, por lo tanto, nuestra hipótesis de partida es que desempeñan un papel importante en la transmisión de ideologías y valores por sus propias características divulgativas y didácticas, así como en la normalización de los usos lingüísticos.

Partiendo de esta hipótesis, proponemos el análisis de los contenidos presentes en las CSL, así como el balance de su repercusión en los espacios digitales con el fin de poner de manifiesto los conceptos y las ideologías lingüísticas vehiculados referentes a la lengua y a las mujeres, y así, comprobar su nivel de aceptación por parte del hablante. Además, este fenómeno actual nos puede brindar más aperturas investigadoras sobre la propia situación de la feminización de la lengua en la sociedad, sobre las CSL como género periodístico y sobre las redes sociales como espacio mediático de debate y difusión de opiniones.

Para ello, en un primer momento, nos acercamos al peculiar género periodístico que son las CSL y al fenómeno lingüístico de feminización del lenguaje. Seguidamente, conformamos el corpus de análisis, formado por todos los artículos publicados desde finales de 2017 hasta principios de 2019 acerca de la feminización de la lengua francesa y más especialmente del français inclusif, en la subsección Langue française de la sección Culture de lefigaro.fr y, más concretamente, en la columna L'Actu des mots (cf. Anexo1).

El contexto político en el que se producen los artículos es relevante en cuanto a la multiplicación de opiniones mediáticas y porque coincide con la publicación de la Circulaire 
du 21 novembre $2017^{1}$ en la que el primer ministro francés Philippe se pronuncia a favor de la aplicación de unos principios de redacción uniforme en los textos oficiales en un intento de legislación de los usos de la lengua en lo que atañe al vocabulario de los nombres de oficio y funciones empleado por la Administración pública.

En este contexto de reconsideración de un sistema lingüístico estrechamente ligado a nociones fundamentales como el sexismo lingüístico, la posición de la mujer en la sociedad o el feminismo nos parece de gran interés reafirmar el vínculo entre lengua y sociedad, demostrando los límites del français inclusif, y más particularmente de la escritura inclusiva por medio de la doble flexión parcial así como la necesidad de una planificación lingüística, $\mathrm{y}$, finalmente, resaltar la importancia del género opinativo que es la CSL para la transmisión de ideas y valores, incrementada con la era digital en la que vivimos, gracias al feed-back que se produce en la redes sociales como Twitter.

\section{Columnas sobre la lengua (CSL) y Chroniques de langage (CDL): caracterización y funciones}

Las CSL, denominadas chroniques de langage en el ámbito francófono $(C D L)$ y objeto del presente trabajo ${ }^{2}$, son representativas de un periodismo especializado y de una tradición discursiva metalingüística indudable poco estudiada hasta hoy en día. En efecto, la abundancia de textos divulgativos de ideas lingüísticas a través de la prensa contrasta con su escaso estudio. En los años setenta, las $C D L$ fueron objeto de las primeras investigaciones en el mundo francófono (Francia y Quebec) con investigaciones como las de Quemada (19701972) o Remysen (2009). En América del Norte y del Sur, pero también en España y otros países europeos los debates sobre la calidad de la lengua también se manifestaron a partir de la segunda mitad del siglo XIX. Hemos de subrayar que si en español, su denominación aún no está afianzada, contamos con una reciente y consecuente propuesta de Marimón Llorca que apoyamos, porque como ella y los autores de varios trabajos que reúne el volumen que edita, "consideramos, pues, que la denominación Columna sobre la lengua para designar este tipo de textos recoge sus rasgos identificadores" (2019: 14).

Este subgénero de opinión cuyos textos son redactados por expertos y no especialmente por lingüistas comparte muchos aspectos con el columnismo: la autoría, la libertad de expresión, su carácter argumentativo y su periodicidad. Pero se diferencia esencialmente en su propósito, pues, su enfoque es la lengua y más concretamente la evolución de la lengua en sociedad. Como indica Santamaría Pérez, "las lenguas, por naturaleza cambiantes, reflejan las transformaciones sociales y culturales que se van produciendo en la sociedad que las

1 Texto en línea: $<$ https://www.legifrance.gouv.fr/jorf/id/JORFTEXT000036068906> [11/08/21].

2 De aquí en adelante, usaremos el término francés Chroniques de langage $(C D L)$ para referirnos al objeto de este estudio ya que nos centramos en las columnas francesas L'Actu des mots, excepto cuando citaremos a autores hispanohablantes y cuando presentaremos peculiaridades de ambas en el punto 4.1. 
habla" (2019: 232). Su presencia coincide con la consciencia lingüística marcada por una cierta inseguridad propia de su comunidad hablante, por razones históricas, patrióticas y por considerar la lengua una herramienta de identificación, de unión y de honor. Por ello, las $C D L$ constituyen un claro fundamento del imaginario lingüístico de los ciudadanos, es decir de la relación que mantienen entre su lengua y el uso que hacen de ella. Su análisis representa un verdadero fenómeno socio-lingüístico, subrayado por Remysen:

Les chroniques de langage représentent donc un phénomène sociolinguistique important -du reste révélateur des rapports particuliers que les francophones entretiennent avec leur langue- qu'il est intéressant d'analyser de plus près. (2009: 2)

Además, tal y como lo considera Quemada, el primer lingüista en poner de manifiesto el interés que supone el estudio de este discurso, las $C D L$ son verdaderos testimonios exclusivos sobre la pureza y la estética de la lengua, e incluso sobre la definición o la preservación del buen uso que, ofrecen datos significativos sobre la evolución de los principales aspectos de la lengua del momento:

[Les chroniques de langage] représentent $[\ldots]$ une somme de témoignages privilégiés pour ce qui touche à la 'pureté' et à l'esthétique de la langue, et plus généralement, pour la définition ou la préservation de tel ou tel 'bon usage' [...] Le fait mérite [ ... ] une attention des plus scrupuleuses puisque, si le contenu des articles ne présente pas un égal intérêt (ils peuvent même en être tout à fait dépourvus), les questions abordées sont en revanche toujours révélatrices de situations propres au français du moment. Considérées dans leur ensemble, elles apportent des données significatives sur l'évolution des principaux aspects de la langue d'aujourd'hui. (1970-1972, vol. 1: i)

Las $C D L$ deben entonces su nombre al espacio en el que se enmarcan: columna. Tanto en la prensa, generalmente escrita, en papel o en línea, en blogs, revistas u otros formatos audiovisuales, estos artículos argumentativos dedicados al análisis de la lengua aparecen redactados por una misma persona, y publicados de manera asidua; además, plantean cuestiones polémicas sobre complejidades de usos lingüísticos, lo que les da ineludiblemente un carácter normativo. Su contribución a la codificación y a la estandarización de la lengua pone en evidencia el desajuste entre la lengua hablada de todos los días y la lengua normativa recogida en manuales prescriptivos. Para Cellard, las $C D L$ son como un instrumento activo de producción y perpetuación de la norma:

Les rapports entre la norme linguistique et ce que l'on nomme génériquement les 'Chroniques de langage' paraissent évidents. En premier lieu, ces chroniques seraient ou auraient été des instruments actifs de production et de perpétuation de la norme; plus rarement de son évolution; exceptionnellement, de sa subversion. En second lieu, le moyen privilégié de sa diffusion. Et en troisième, par l'intérêt qu'elles suscitent en amont (questions posées au chroniqueur) et en aval (réactions des lecteurs aux chroni- 
ques), un bon indice du degré et de la nature des préoccupations du public à l'égard de cette norme ${ }^{3}$. (1983: 651)

A través de la opinión del columnista, la $C D L$ presenta una interpretación de la realidad que orienta al lector quien, de manera general busca respuestas. Este espacio se transforma en una plataforma para exhibir su pensamiento e incluso su tendencia política y reforzar el vínculo privilegiado entre el columnista y el lector. Según Morán Torres, existe:

Una coherencia previa entre la línea ideológica o cultural del periódico y la del articulista que se sirve de sus páginas como vehículo de sus ideas. La falta de coincidencia general entre las posiciones del medio y las del columnista daría lugar a un desconcierto público. (1988: 167)

Esta consideración nos permite poner de relieve la importancia del ethos del columnista quien actúa como un divulgador de su ideología lingüística. Su influencia invita a los lectores a reflexionar sobre su actividad lingüística y a poner en tela de juicio sus propios usos y los de otros miembros de su comunidad de habla. De esta manera, las $C D L$ consiguen llegar al lector no especializado, y es en parte gracias a la situación de poder en la que se encuentra el columnista y su reconocimiento social porque, como subraya López Pan, su ethos "se convierte en un banderín de enganche" (1995: 25). Para Grijelmo, estos discursos son únicos porque son instructivos y pedagógicos. Representan una verdadera oportunidad para el ser humano y la sociedad en general porque su construcción pasa por el idioma que considera como un elemento unificador e identitario que ayuda a entender mejor el pasado y a enfocar mejor el futuro. En Intervención en Cartagena de Indias, considera las CDL una "suerte de periodismo cultural que consiste en difundir las reglas de nuestra lengua con la destreza puesta en que el lector más desavisado las entienda” (2007: 2).

Analizaremos por lo tanto las $C D L$ desde esta caracterización y siguiendo la completa y reciente definición propuesta por Sánchez Manzanares:

La columna sobre la lengua es un discurso que un autor publica periódicamente en la prensa para abordar cuestiones lingüísticas, principalmente referidas a usos "incorrectos" de una lengua por contraposición a usos prescriptivos o aconsejables. Tiene un propósito divulgativo $\mathrm{y}$, al mismo tiempo, un carácter argumentativo, por cuanto estamos ante un artículo de opinión en el que el autor valora la actuación verbal, ya sea escrita u oral, con relación a su idea de lengua y de lo normativo. (2019: 217-218)

3 La relación entre la norma lingüística y lo que llamamos genéricamente "CSL" parecen evidentes. En primer lugar, estas columnas serían o habrían sido unos instrumentos activos de producción y de perpetuación de la norma; con menos frecuencia de su evolución; excepcionalmente de su subversión. En segundo lugar, el medio privilegiado de su difusión. $\mathrm{Y}$, en tercer lugar, por el interés que suscitan con antelación (preguntas planteadas por el columnista) y con posterioridad (reacciones de los lectores a las crónicas), un buen índice del grado y de la naturaleza de las preocupaciones del público con respecto a esta norma (traducción personal). 


\section{La feminización del lenguaje en el discurso público, tanto oficial como mediático}

\subsection{Lenguaje inclusivo y escritura inclusiva}

En varios países occidentales, el lenguaje inclusivo es una propuesta que consiste en encontrar respuestas a las reivindicaciones feministas por la legitimidad de la mujer y su visibilidad tanto en la sociedad como en la lengua. Erradicar el sexismo social implica inexorablemente erradicar el sexismo lingüístico. El uso genérico del masculino sitúa al hombre y a su punto de vista en el centro de todo lo que existe. Este androcentrismo o sexismo lingüístico discrimina a las mujeres y a otras personas de otras entidades.

El lenguaje inclusivo, también llamado incluyente o no sexista nace de la necesidad de compensar la ausencia de formas femeninas en el lenguaje. El término inclusivo indica justamente que el lenguaje no excluye a nadie, ni por motivo de sexo, de edad, de origen étnico y tampoco de orientación sexual. Usar el lenguaje incluyente consiste, por lo tanto, en acercarse al concepto filosófico de inclusión desde una perspectiva política que permite visibilizar a los sujetos más sensibles. Esta teoría es la base del principio de inclusión en los procedimientos lingüísticos propuestos por Alpheratz:

Cette variété du français standard applique des processus langagiers dont le point commun est d'inclure dans l'ontogénèse [...] les genres sociaux minorisés par l'emploi générique du genre grammatical masculin -lequel prétend exprimer une pensée universelle- et de visibiliser à la fois le principe grammatical excluant (le genre masculin ou emploi générique) et les représentations symboliques ou sociales que cet emploi n'exprime pas. ${ }^{4}$ (2018: 2-3)

Asimismo, la lingüista define el français inclusif como:

[...] l'ensemble des processus langagiers qui s'écartent du français standard, fondés en général sur la notion de genre, et en particulier sur le rejet d'une hiérarchie entre les représentations symboliques et sociales associées aux genres grammaticaux, ces variations ayant pour objectif d'inclure et de visibiliser tous les genres dans la langue comme dans la pensée ${ }^{5}$. (2008: 3$)$

4 Esta variedad del francés estándar aplica procedimientos lingüísticos cuyo punto en común es incluir dentro de la ontogenia [...] los géneros sociales minorizados por el empleo genérico del género gramatical masculino -el cual pretende expresar un pensamiento universal- y visibilizar a la vez el principio gramatical excluyente (el masculino genérico o empleo genérico) y las representaciones simbólicas o sociales que este uso no expresa (traducción personal).

5 [...] el conjunto de procedimientos lingüísticos que se alejan del francés estándar, fundados en general sobre la noción de género, y en particular sobre el rechazo de una jerarquía entre las representaciones simbólicas y sociales asociadas a los géneros gramaticales, estas variaciones teniendo como objetivo incluir y visibilizar todos los géneros dentro de la lengua como dentro del pensamiento (traducción personal). 
La inclusividad que da lugar al proceso de feminización del lenguaje pone en relación la consciencia de género con la identidad, la paridad y la perfomatividad de la lengua. Así es como define Alpheratz el concepto de variación diaética, variation diaéthique, como respuesta a la materialización de esta variedad del francés estándar: "Sa définition peut être une variation relevant de la conscience de genre, d'identité, d'égalité et de la performativité de la langue" (2018: 7).

Como ella, consideramos esta variación un punto de partida a la feminización de la lengua y más concretamente al français inclusif cuya formación se establece mediante varios procedimientos lingüísticos que pueden usarse de manera aislada o combinada: 1) Regla de proximidad y sufijación, 2) Género neutro, 3) Reactivación de palabras o de regularidades antiguamente atestadas, 4) Epicenización, 5) Escritura inclusiva por medio de la doble flexión total o parcial, 6) Hipernomización, 7) Neologismos y 8) Buen uso de la lengua.

En nuestro estudio, nos centraremos en el procedimiento 5) relativo a la escritura inclusiva por medio de la doble flexión total o parcial que reúne dos estrategias en una ${ }^{6}$. La primera se caracteriza por retomar la palabra entera flexionada en un género diferente del masculino. En este caso, el orden alfabético debe prevalecer. Esta estrategia abunda en las alocuciones políticas, recordemos el principio de los discursos del General de Gaule siempre encabezados por: Françaises, Français. La segunda tiene como peculiaridad la inserción de marcas morfológicas de flexión. Un ejemplo de doble flexión parcial podría ser el título de un artículo del periódico francés Libération "Prêt•e•es à utiliser l'écriture inclusive?" del 27 de septiembre de 2017 con la inserción del point médian o punto medio.

Es justamente, la doble flexión parcial que el primer ministro Philippe quiere eliminar de todo documento administrativo porque presenta muchas controversias. Alpheratz la define como:

[...] un processus langagier consistant à visibiliser la coprésence des genres sociaux dans l'écriture en ajoutant une marque morphologique de genre autre que celle du masculin à un mot déjà fléchi à ce genre et utilisé en emploi générique. L'écriture inclusive signale cette marque par l'insertion de signes ou de formats typographiques (point, point médian, tiret, parenthèses, barre oblique, capitale) $)^{8}$. (2018: 9)

De estas marcas tipográficas y morfológicas, resaltamos: 1) la inserción del point médian o punto medio, que tanto debate origina en la actualidad por su difícil acceso en muchos

6 Alpheratz (2018) distingue la escritura inclusiva o doble flexión parcial de la doble flexión total, pero no consideramos esta distinción muy relevante y preferimos utilizar el concepto de escritura inclusiva de manera más global, es decir que incluimos en escritura inclusiva los dos fenómenos lingüísticos.

7 Ver: $<$ https://www.liberation.fr/france/2017/09/27/pretes-a-utiliser-l-ecriture-inclusive_1598867/> [02/08/2021].

$8[\ldots]$ un proceso lingüístico que consiste en visibilizar la copresencia de los géneros sociales en la escritura añadiendo una marca morfológica de género diferente de la del masculino a una palabra ya flexionada en este género y utilizada en un empleo genérico. La escritura inclusiva señala esta marca mediante la inserción de signos o formatos tipográficos (punto, punto medio, guion, paréntesis, barras oblicuas, mayúsculas) (traducción personal). 
teclados ${ }^{9}$ todavía como por su ilegibilidad para las personas con incapacidad visual, 2) el uso de paréntesis de connotación relativamente excluyente porque lo que se pone entre paréntesis es considerado de menor relevancia, una connotación también vehiculada por la expresión mettre entre parenthèses que significa "descuidar, excluir, hacer abstracción de"10, 3) las barras oblicuas que implican una oposición y que, junto con los paréntesis, parecen sobrecargar el texto, 4) el guion que presenta problemas de confusión con las palabras compuestas, 5) el punto que plantea problemas con la puntuación, y finalmente 6) el uso de alternativas ajenas al sistema de nuestra lengua (@, x, e) para dar cabida a la diversidad sexual que no se identifica con la dualidad masculino/femenino y que resultan polémicas.

Para los defensores de la escritura inclusiva por medio de la doble flexión parcial, su uso permite una economía de palabras, pero Alpheratz (2018) pone en evidencia la controversia que genera, pues, presenta igualmente un problema de economía lingüística al emplear más unidades: marcas morfológicas y signos tipográficos. Además, una opinión recurrente acerca de la doble flexión parcial es que dificulta lectura y que es difícilmente aplicable al discurso oral.

\subsection{Debate lingüístico y social de la feminización del lenguaje}

El lenguaje es un claro reflejo de la sociedad en la que vivimos y condiciona nuestra percepción del mundo, como subraya Luque Durán: “Toda lengua es un mapa o retrato parcial e inconsciente del mundo y no existe isomorfismo entre lengua y mundo" (2004: 491). Según sus estudios sobre lenguaje y cognición basados en investigaciones de grandes lingüistas como Boas, Sapir y Worf, los hablantes tienen diferentes visiones del mundo en función de la sociedad a la que pertenecen y que

es obvio que, para comprender bien una lengua, tanto su gramática y su léxico como sus usos pragmáticos es necesario comprender su cultura, es decir los valores sociales y culturales asociados a las palabras. (2004: 492)

Los cambios lingüísticos que supone el lenguaje inclusivo están estrechamente ligados a los cambios sociológicos. En España, se establecieron durante la Transición en los años 80 junto con la creación del Instituto de la Mujer (1983) y el inicio de abundantes y fecundas investigaciones lingüísticas y guías sobre el lenguaje no sexista. En el mundo francófono, cada comunidad ha recorrido su propio camino hacia la adopción o vuelta a las apelaciones femeninas, en un primer momento de oficios eméritos. Quebec, considerada zona precursora

9 La Asociación francesa de normalización (AFNOR) está trabajando, desde 2017, para incluir esta opción y de manera general por considerar la escritura inclusiva en los teclados franceses en una nueva norma. Ver: $<$ L'écriture inclusive, une option retenue dans la future norme volontaire sur le clavier français - AFNOR Normalisation $>[02 / 05 / 21]$.

10 Ver: <PARENTHÈSE: Définition de PARENTHÈSE (cnrtl.fr) $>$ [02/07/21]. 
en lo que concierne la feminización del lenguaje y la eliminación del sexismo lingüístico empezó su proceso hace treinta años gracias a un importante movimiento social y popular que fue seguido por varias publicaciones tales como: Pour les Québécoises: égalité et indépendance (1978), Au féminin, Guide de féminisation des titres de fonction et des textes (1991) y Formation sur la rédaction épicène. Además, se llevaron a cabo acciones planificadoras por parte de l'Office québécois de la langue française. En Bélgica y Suiza, la cuestión se planteó en 1993 a través de decretos acompañados de otras publicaciones, en particular guías.

En Francia, este proceso fue menos inherente y más artificial puesto que se trata más bien de una reconquista perdida durante siglos iniciada. Numerosos estudios demostraron que hasta el siglo XVI, la lengua contaba con formas lexicales femeninas correspondientes a oficios, títulos, cargos y funciones. Tal y como lo admiten Mathieu y Pierrel, en todos los niveles de la escala social, las mujeres eran visibles: "du haut au bas de l'échelle sociale, les femmes étaient présentes et leurs activités énoncées par des termes qui rendaient compte de leur sexe" (2009: 112). Términos como autrice y écrivaine no suscitaban tanta controversia como ahora y su presencia en la lengua reflejaba realidades sociológicas tan relevantes como el simple acceso de las mujeres al mundo laboral mayormente ocupado por hombres. Pero a partir del siglo XVIII, marcado por una política centralista que usaba la lengua como bandera de unión social y nacional haciéndola menos rica y flexible, se vuelve a masculinizar, apartando así a las mujeres de las profesiones literarias, de la reflexión sobre el lenguaje, y finalmente de las profesiones eminentes.

En la lengua francesa, hasta el siglo XIX, asistimos a lo que llama Cerquiglini (2019) el Imperio del femenino conyugal devolviendo la mujer a la posición de la "esposa de", es decir que, por ejemplo, ya no era ambassadrice sino la femme de l'ambassadeur. A partir de este siglo, la lucha por la visibilidad de la mujer en la lengua se retomó y se sigue llevando a cabo paulatinamente respondiendo a la necesidad de reflejar la evolución de la sociedad, de las mentalidades y por consiguiente de una lengua mundial ${ }^{11}$ que se quiere dinámica gracias a diversas acciones estatales. Entre ellas, podemos citar la creación en 1984 de una comisión de terminología, presidida por Groult, legislada mediante el Decreto n83-153 del 29 de febrero, cuyos resultados fueron publicados dos años más tarde en la Circulaire du 11 mars 1986 relative à la féminisation des noms des métiers, fonction, grade ou titre. El Decreto del 17 de diciembre de 1997 sorprende y nombra a una directrice ${ }^{12}$ del personal docente. Un año más tarde, se publicó la Circulaire du 6 mars 1998 relative à la féminisation des noms des métiers, fonction, grade ou titre solicitando investigaciones por parte de la Comission Générale de Terminologíe et Néologie ${ }^{13}$ y se publicó la guía Femme, j’écris ton nom .... guide

11 Para el actual Ministro de la Educación nacional Blanquer "Notre langue est le premier trésor français, celui qui nous relie tous et fait notre puissance mondiale". Entrevsita en LeFigaro.fr el 3 de mayo de 2021, ver: $<$ Jean-Michel Blanquer veut interdire l'écriture inclusive à l'école - Le Figaro Etudiant> [03/05/21].

12 Ver: $<$ https://www.legifrance.gouv.fr/jorf/id/JORFTEXT000000187072> [02/08/21].

13 Actualmente, la comisión se llama La Commission d'Enrichissement de la langue française. 
d'aide à la féminisation des noms de métiers, titres, grades et fonctions (Becker y al., 1999). En 2013, se creó Le Haut Conseil à l'égalité entre les femmes et les hommes, una autoridad de reflexión, evaluación y proposición de política sobre los derechos de la mujer y la paridad hombre-mujer a nivel político, económico, cultural y social. En 2017, el primer ministro Philippe firmó la Circulaire du 21 novembre 2017 relative aux règles de féminisation et de rédaction des textes publiés au Journal officiel de la République française a favor de la aplicación de unos principios de redacción uniforme en los textos oficiales.

Este documento gubernamental reavivó el debate sobre la feminización de la lengua en todos sus aspectos y, a partir de su publicación, las $C D L$ intensificaron sus artículos de opinión también mediante entrevistas realizadas a especialistas de este campo. Las divergencias de opiniones sitúan a la comunidad hablante en una situación abrumadora por los múltiples debates que surgen en todos los medios y por la falta de uniformidad convencional. Desde hace siglos, nos seguimos encontrando con discursos opuestos, unos más convencionales y puristas frente a otros más abiertos e innovadores. Los actores de divulgación de la variación lingüística tienen un cometido muy importante, pero siempre se ha impuesto en las mentes que la decisión última sobre el uso correcto o incorrecto, la proporcionan los diccionarios y las gramáticas. En el caso del español, es la RAE la institución que desde 1973 sujeta el castellano a prescripciones y dictámenes. En Francia, es la Académie Française $(A F)$ creada en 1635 por Richelieu, en pleno siglo de oro de la monarquía absoluta del siglo XVII, que implantó una ideología unilingüística y fetichista. Ambas instituciones, aferradas a la tradición, representan la mayor oposición a la feminización del lenguaje pues, tienen una autoridad normativa que, en ocasiones, obstaculiza la posible entrada de variaciones lingüísticas. Boyer (2012), haciendo referencia a las denuncias de Bourdieu y Boltanski (1975), estima que este ideal lingüístico provoca que toda aparición nueva sea considerada como una amenaza a la perfección de la lengua y por lo tanto rechazada y estigmatizada: "Dès lors tout ce qui va apparaître comme une menace contre la perfection de la langue sera a priori refusé, rejeté, stigmatisé" (2012: 3).

Ahora bien, no podemos obviar que el alcance intencionado de la escritura inclusiva es indudablemente político y democrático. En efecto, Para Baurens, "l'état démocratique doit avoir pour objectif de réduire l'exclusion, en particulier celle des femmes"14 (2019: 3) y Hugonnier (2019: 3) afirma que "avant d'être une affaire de linguistique, l'application de l'écriture inclusive est d'abord un geste politique"15. La legitimización de este componente del francés inclusivo se desarrolla en un campo de batalla donde la lengua es protagonista de una lucha ideológica entre tradicionales, innovadores, y moderados, especialistas o no especialistas, en espacios privados, pero sobre todo públicos. Las diversas medidas tomadas

14 El estado democrático debe tener como objetivo reducir la exclusión, en particular la de las mujeres (traducción personal).

15 Antes de ser un asunto de tipo lingüístico, la aplicación de la escritura inclusive es ante todo un gesto político (traducción personal). 
por los gobiernos a nivel internacional sugiriendo el uso de un lenguaje igualitario, suscitan un intenso debate que, siguiendo la reflexión de Guerrero Salazar, es "de gran repercusión en los medios de comunicación, que sigue vigente en la actualidad, donde ha cobrado fuerza gracias a las redes sociales" (2020: 204).

En nuestra sociedad, el cuarto poder, es decir, los medios de comunicación de masas son determinantes en la formación de la opinión pública. Internet, la Web 2.0, la comunicación móvil y una pluralidad de herramientas de software social junto con la emergencia de la autocomunicación los están modificando, ofreciendo un abanico comunicativo que permite una mayor intervención de los ciudadanos. Se trata de autocomunicación porque según Castells:

uno mismo genera el mensaje, define los posibles receptores y selecciona los mensajes concretos o los contenidos de la web y de las redes de comunicación electrónica que quiere recuperar. (2009: 81)

El uso de la inteligencia artificial en las redes sociales y otras plataformas digitales, los ya famosos "algoritmos" de Facebook, Twitter o Google, permiten a cada cual decidir qué leer y qué no leer. La comunicación deja de ser unidireccional y pasa a ser horizontal. El desarrollo de redes horizontales de comunicación interactiva incluye el intercambio multimodal de mensajes interactivos de muchos a muchos, tanto sincrónicos como asincrónicos.

Para dar cuenta de la calidad de transmisora de valores e ideologías de las $C D L$, queremos dar cuenta de su repercusión en el lector a través de su participación en la plataforma Twitter porque es una fuente de poder e influencia social muy representativa de esta revolución tecnológica y presenta características propias que resultan muy pertinentes para el estudio de la lengua. En efecto, gracias a ella, Gutiérrez-Rubí afirma que se consigue transmitir "una sensación de proximidad y de igualdad que, en la red, es posible y altamente valorada" (2008: 80). Sus peculiares funcionalidades, llamadas "activités technoscripturales"16 por Paveau (2013: 9) permiten redactar un mensaje (un tweet) de no más de 140 caracteres, compartir un tweet (retwitear), publicar un video en directo (livetwitear) y enviar mensajes privados o DM (Direct Message) a sus amigos (followers). Creado en 2006, esta red social gratuita de microblogging o nanoblogging es una microesfera, también llamada "Twitosfera", que permite seguir a personas, comercios, entidades o hilos temáticos gracias a su particular etiqueta "hashtag" como palabra clave para la búsqueda. Para Mancera Rueda y Pano Alemán, en este tipo de red,

[...] lo que se pretende es más bien informar, informarse y opinar sobre cualquier tema compartido por los usuarios que se siguen, generalmente entidades públicas y privadas, celebridades y expertos en todo tipo de cuestiones que, en ocasiones, se erigen en líderes de opinión. (2014: 58)

16 Actividades tecnoscripturales (traducción personal). 
Con más de 300 millones de usuarios en el mundo, esta red permite convertir ciertos temas como "trending topic" a través de sus diversas funcionalidades, temas compartidos por personas que comparten los mismos intereses.

\section{Análisis des $C D L$ de Le Figaro}

\subsection{Contextualización y metodología}

El corpus está formado por 23 artículos publicados en la versión digital del periódico Le Figaro en la CDL L'Actu des mots de la subsección Langue française de la sección Culture. Para seleccionarlos, hemos atendido en un primer momento varios criterios como su temática, su periodicidad y su naturaleza siendo todos artículos de opinión con carácter divulgativo y función didáctica sobre cuestiones de lenguaje. La sección Langue française cuenta con varios espacios relacionadas con la lengua francesa, también en formato de foro, tests, ejercicios ortográficos, buscadores léxicos y de conjugación: Forum, Expressions, Francophonie, Testez vos connaissances, La Dictée, Dictionnaire, Le Conjugeur. De todas ellas, L'Actu des mots es el espacio fijo que ofrece más contenidos relacionados con la cuestión de la feminización de la lengua.

Para proceder a la recopilación del corpus, nos basamos en un primer momento en la temática de la CDL L'Actu des mots, pues nos interesaba recopilar e investigar en un primer momento todos los contenidos que tratasen de la feminización de la lengua. Después, tomamos en cuenta el criterio correspondiente a la firma, un elemento propio de este género periodístico, es decir, el autor y su asiduidad de publicación. Como en esta $C D L$ muchos periodistas redactan sobre diversos temas, consideramos más congruente formar el corpus a partir de varios de ellos, que presentamos más adelante, con el fin de obtener un abanico más amplio de opiniones.

En cuanto a la datación, la lectura de las $C D L$ seleccionadas abarca un periodo de tiempo de un año y tres meses, siendo el elemento detonante de un intenso debate en Francia la Circulaire du 21 novembre 2017 en la que el primer ministro francés, Philippe, dio instrucción a sus ministros de proscribir la escritura inclusiva por medio de la doble flexión parcial en los documentos oficiales alegando que:

[...] outre le respect du formalisme propre aux actes de nature juridique, les administrations relevant de l'État doivent se conformer aux règles grammaticales et syntaxiques, notamment pour des raisons d'intelligibilité et de clarté de la norme ${ }^{17}$.

17 Además del respeto del formalismo propio de los actos jurídicos, las administraciones sujetas al Estado deben atenerse a las reglas gramaticales y sintácticas, especialmente por razones de inteligibilidad y de claridad de la norma (traducción personal). 
En este documento reglamentario, se quiere legislar los usos de la lengua en lo que atañe al vocabulario de los nombres de oficio y funciones empleado por la Administración pública. Este punto de partida nos parece de gran importancia porque aparte de reavivar un debate sociolingüístico latente en una época de reconsideración del sistema lingüístico, de cuestionamiento de las autoridades y de los organismos "guardianes" de la lengua francesa y de replanteamiento de los valores sociales, pone de manifiesto un rechazo hacia una estrategia lingüística en concreto: la escritura inclusiva por medio de la doble flexión parcial. Sin embargo, detalla más prácticas recomendadas para la redacción de documentos como el uso de la escritura inclusiva por medio de la doble flexión total, así como el uso del masculino genérico, la concordancia nominal y la feminización de los nombres de oficio. En esta situación de dudas y controversia, el 28 de febrero 2019, el Conseil d'État le daba la razón al gobierno ${ }^{18}$ rechazando las demandas que pedían la anulación de esta circular y alegando que, al estar destinada exclusivamente a los miembros del gobierno y al personal de la Administración pública, no puede "être regardée comme ayant pour objet ou pour effet de porter atteinte à l'égalité entre les femmes et les hommes"19.

El corpus analizado presenta características propias porque la CDL L'Actu des mots respeta ciertas propiedades inherentes a este género como la continuidad, periodicidad o asiduidad de sus publicaciones y su posición fija en el periódico que resulta ser un referente visual y fiel para el lector.

En contraparte, por lo que atañe a la firma, hemos de subrayar que a la diferencia de lo que ocurre con las CSL en la prensa española, en lefigaro.fr hay columnas con título fijo que son publicadas periódicamente y que no siempre están firmadas por el mismo autor. Nos encontramos con 20 columnas de autor y 3 columnas de tema firmadas por varios autores. Además, de las 20 columnas de autor, 4 vienen firmadas por lefigaro.fr, por $A F P^{20}$, es decir por la editorial sin precisar si por un mismo periodista. El siguiente parámetro, tiene que ver con la tipología de casi la mitad de los textos que constituyen el corpus de este trabajo: 11 de ellos son entrevistas de tipo opinante realizadas por columnistas a especialistas. Por lo tanto, la opinión destacable resulta ser la de la persona entrevistada ya que los columnistas se limitan a hacer las preguntas.

Así pues, queremos dejar claro que L'Actu des mots funciona como una $C D L$ de forma particular porque las entrevistas que incluye constituyen un género periodístico distinto a un artículo de opinión, que es lo en lo que consiste básicamente una columna. En las entrevistas, el periodista fija un tema lingüístico durante la entrevista para que quede expuesta la opinión del entrevistado y es lo que le confiere la misma finalidad que una $C D L$. Por tanto,

18 Ver: $<$ https://www.legifrance.gouv.fr/ceta/id/CETATEXT000038186296> [02/08/21].

19 No puede ser observada como algo cuyo objeto y efecto vulneraría la paridad entre mujeres y hombres (traducción personal).

20 Agence France-Presse: Agencia de noticias más antiguas del mundo. Tiene su sede en París y más de cien oficinas en el mundo. Según su estatuto, tiene por misión investigar tanto en Francia como en el extranjero los elementos de información completa y objetiva y de ponerla a la disposición del usuario. 
concebimos que L'Actu des mots es una $C D L$ desde el punto de vista de la función que cumple porque valoramos que incluye textos metalingüísticos con función didáctica y carácter argumentativo.

Asimismo, nos parece importante hacer hincapié en otro aspecto actual que ha transformado el formato en el que se inscribe este género. En efecto, Internet y los nuevos formatos hacen difícil una clasificación hermética de las $C D L$, pues se han vuelto dinámicas e interactivas. El formato digital cuenta con una riqueza de recursos no presentes en una versión en papel. Gracias a su configuración, la columna dispone de enlaces relacionados con el tema, ofrece la posibilidad de acceder a otras secciones en un clic e incluso de ver vídeos incrustados que amplían los contenidos tratados aportando más información aún. Por añadidura, permite a sus lectores realizar comentarios en línea y compartir en las redes sociales. Estos dos últimos parámetros representan marcadores interesantes para nuestro estudio puesto que dan protagonismo a la comunidad lingüística y nos permiten estimar la repercusión de los contenidos de las $C D L$ en ella.

Este alcance pasa por las opiniones de los columnistas y especialistas vertidas en las $C D L$ y por lo tanto por sus propias ideologías sobre el tema. Su firma, proveniente de entidades importantes estrechamente relacionadas con la lengua francesa ( $A F$, Le Petit Robert, por ejemplo) representan una fuerza innegable porque contribuyen a la creación de una relación íntima y de confianza con el lector. Los columnistas y entrevistados estudiados en este corpus son los siguientes:

- Columnistas.

Alice Develey: periodista literaria en lefigaro.fr desde el 4 de enero de 2016. Tiene 1359 artículos publicados en este periódico. Analizaremos 15 artículos suyos, más de la mitad de la totalidad del corpus, y 3 que comparte con Claire Conruyt.

Claire Conruyt: periodista especializada en Lengua francesa y literatura en lefigaro.fr desde el 5 de mayo de 2018. Tiene 147 artículos publicados en este periódico. Aparte de los 3 artículos en común con Alice Develey, analizaremos 1 más de su firma.

Marie-Estelle Pech: periodista especializada en Educación en lefigaro.fr desde el 03 de febrero de 2010. Tiene 1395 artículos publicados en este periódico. Analizaremos 1 artículo firmado por ella.

Jean Talabot: periodista en lefigaro.fr desde el 08 de agosto de 2016. Tiene 826 artículos publicados en este periódico. Analizaremos 1 artículo firmado por él.

Jean Pruvost: lexicólogo y periodista en lefigaro.fr desde el 17 de marzo de 2017. Tiene 79 artículos publicados en este periódico. Analizaremos 1 artículo firmado por él. Por último, analizaremos 4 artículos firmados por lefigaro.fr.

- Entrevistados.

Marie-Dominique Porée: profesora de gramática, griego y especialista en literatura latina. Autora de Olympe de Gouges et autres femmes révolutionnaires (2019) y otras obras relacionadas con la lingüística francesa, el griego y el latín. 
Eliane Viennot: lingüista, historiadora de la literatura y crítica literaria. Profesora de literatura medieval.

Bernard Cerquiglini: lingüista especializado en la historia de la lengua francesa. Ha publicado un libro sobre la feminización de los nombres de oficio: Le Ministre est enceinte ou la grande querelle de la féminisation des noms (2018).

Jeanne Bordeau: Estilista del lenguaje, fundadora del Instituto de la calidad de expresión $^{21}$.

Loïc Depecker: delegado general de la lengua francesa $\left(D G L F L F^{22}\right)$ desde mayo de 2015. Es un lingüista francés especializado en terminología y lexicología.

Alain Rey: lingüista, lexicógrafo y cofundador del Petit Robert.

Frédéric Vitoux: escritor, crítico y académico.

Danièle Sallenave: académica, miembro de la $A F$ y de la Comisión encargada de estudiar la feminización de la lengua en el seno de la $A F$.

Dominique Bona: escritora francesa, miembro de la $A F$ desde 2013 miembro de la Comisión encargada de estudiar la feminización de la lengua en el seno de la $A F$.

La tabla que sigue nos ofrece informaciones acerca del número de artículos analizados en función de su tipo ( $C D L$ o entrevista) y del tiempo (año de publicación) $)^{23}$.

\begin{tabular}{|c|c|c|c|c|}
\hline Criterios & Número de artículos & 2019 & 2018 & 2017 \\
\hline Alice Develey & 12 & 3 & 2 & 7 \\
\hline Entrevista & 8 & & & \\
\hline$C D L$ & 4 & & & \\
\hline Alice Develey y Claire Conruyt & 3 & 3 & & \\
\hline Entrevista & 2 & & & \\
\hline$C D L$ & 1 & & & \\
\hline Claire Conruyt & 1 & 1 & & \\
\hline Entrevista & 1 & & & \\
\hline$C D L$ & 1 & & & \\
\hline Le Figaro & 4 & 2 & & 2 \\
\hline$C D L$ & 4 & & & \\
\hline Marie Estelle Pech & 1 & & & 1 \\
\hline$C D L$ & 1 & & & \\
\hline Jean Talabot & 1 & & & 1 \\
\hline$C D L$ & 1 & & & \\
\hline Jean Pruvost & 1 & & & 1 \\
\hline$C D L$ & 1 & & & \\
\hline
\end{tabular}

Figura 1: Relación periodistas - Número por año y tipo de artículos.

21 Ver: <https://madamelangage.com> [10/08/21].

22 Délégation Générale à la Langue Française et aux Langues de France, ver: <http://www.culture.gouv.fr/Thematiques/Langue-francaise-et-langues-de-France/La-DGLFLF> [02/09/19].

23 La tabla en Anexo nos proporciona más información sobre el corpus. 


\subsection{Análisis del corpus y resultados}

El primer resultado más general y sugerente es la presencia de artículos sobre este fenómeno socio-lingüístico en el tiempo. Como lo hemos señalado anteriormente, en 2017, cuando se publicó la Circulaire del primer ministro Philippe, es decir del 21 de noviembre al 31 de diciembre, abundaron los artículos de opinión al igual que en 2019, cuando se publicó, el 28 de febrero, un informe favorable a la feminización de la lengua. En la tabla siguiente, podemos comprobar que el número de publicaciones sobre la feminización del lenguaje es mucho más elevado en esos dos años que en 2018, lo que revela el alto grado de interés por este tema lingüístico acorde con la publicación de ambos documentos gubernamentales.

\begin{tabular}{lc}
\hline Total artículos & 23 \\
\hline Total 2019 & 9 \\
\hline Total 2018 & 2 \\
\hline Total 2017 & 12 \\
\hline
\end{tabular}

Figura 2: Número de artículos por año.

En segundo lugar, la lectura del corpus nos revela una presencia escasa de procedimientos como la epicenización, la hiperonimisación y el buen uso de la lengua, y comprobamos que los procedimientos lingüísticos relacionados con la escritura inclusiva (doble flexión total y parcial), la reactivación de palabras o de regularidades antiguamente atestadas, el género neutro, los neologismos y la regla de proximidad y sufijación, son, en este orden de relevancia los más comentados y analizados tanto por los columnistas como por los entrevistados. Como ya mencionamos, existe una interrelación entre todos los procedimientos lingüísticos porque la feminización de la lengua se hace efectiva mediante uno o varios de ellos combinados. En total, registramos 59 menciones a los procesos lingüísticos referidos al français inclusif. Siguiendo estos datos, podemos afirmar que la mayor preocupación de los periodistas y lingüistas o expertos entrevistados, y por ende de la sociedad, por el fenómeno lingüístico relativo a la feminización de la lengua, se focaliza especialmente en dos procesos lingüísticos: la escritura inclusiva y la reactivación de palabras antiguamente atestiguadas, coincidiendo con las voluntades políticas expresadas en la Circulaire du 21 novembre 2017. La escritura inclusiva por medio de la doble flexión total y parcial se repite en 15 ocasiones y la reactivación de palabras o regularidades antiguamente atestiguadas en 11 ocasiones.

\begin{tabular}{lc}
\hline Estrategias lingüisticas & Ocurrencias \\
\hline 1. Regla de proximidad y Sufijación & 8 \\
\hline 2. Género neutro & 10 \\
\hline 3. Reactivación de palabras o de regularidades antiguamente atestadas & 11 \\
\hline 4. Epicenización & 2 \\
\hline
\end{tabular}




\begin{tabular}{lc}
\hline Estrategias lingü̈sticas & Ocurrencias \\
\hline 5. Escritura inclusive: doble flexión total y parcial & 15 \\
\hline 6. Hipernomización & 2 \\
\hline 7. Neologismos & 8 \\
\hline 8. Buen uso de la lengua & 3 \\
\hline TOTAL & 59
\end{tabular}

Figura 3: Tabla de las ocurrencias lingüísticas.

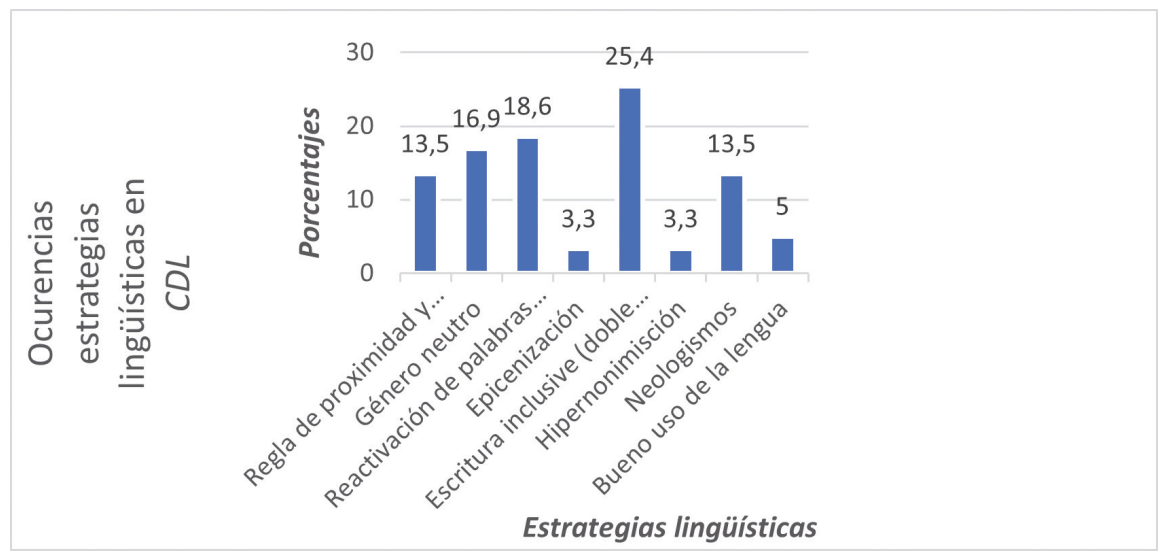

Figura 4: Gráfico de las ocurrencias lingüísticas (\%).

El fenómeno lingüístico que provoca un rechazo casi total y unánime es la escritura inclusiva por medio de la doble flexión parcial. Los argumentos en contra proliferan mientras que no aparece ninguno en contra del uso de la doble flexión total. Las opiniones vertidas sobre este polémico proceso lingüístico ponen de manifiesto su complejidad y falta de igualdad precisamente cuando se quiere ser inclusiva.

\begin{tabular}{|c|c|c|c|}
\hline & \multicolumn{2}{|l|}{ Escritura inclusiva } \\
\hline & & Doble flexión total & Doble flexión parcial \\
\hline & & & - Escritura ilegible \\
\hline & & & - Corta la lectura \\
\hline & & & - Inadaptada a la lengua oral \\
\hline & & & - Disocia la lengua \\
\hline & $\Xi$ & & - Dificulta el aprendizaje \\
\hline & 8 & & - Representa un obstáculo para las personas ciegas y disléxicas \\
\hline$\cong$ & 这 & & - Falta de unificación \\
\hline 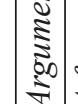 & 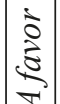 & & - Es más rápida que la doble flexión total \\
\hline
\end{tabular}

Figura 5: Tabla de los argumentos a favor y en contra de la escritura inclusiva. 
Este fenómeno es por lo tanto el que provoca más discordias porque, a pesar de suponer un peligro para la lengua, sus defensores ven en él la mejor forma de luchar contra las desigualdades entre los sexos a partir de la lengua.

En tercer lugar y con el fin de comprobar el nivel de aceptación por la comunidad lingüística de estos fenómenos lingüísticos, hemos atendido las repercusiones de los artículos en base a dos perspectivas: la primera, en los comentarios internos de la $C D L$ y la segunda, en el feed-back de los artículos en Twitter a través de publicaciones y comentarios. De este modo, hemos procedido a dar cuenta la importancia de los fenómenos lingüísticos relativos a la feminización del lenguaje en función de sus proyecciones mediáticas. En efecto, consideramos que, si un artículo da mucho que hablar, es porque su contenido es polémico e interesa a los hablantes. De los 23 artículos analizados, los 15 sobre la escritura inclusiva son los que, de manera global, presentan más retroalimentación no sólo interna sino externamente. Por añadidura, hemos constatado que el artículo que acumula más incidencia tanto interna en la propia $C D L$ como externa en Twitter es el relativo a la publicación de la Circulaire de Philippe sobre la proscripción del uso de la doble flexión parcial en los documentos administrativos Édouard Philippe bannit l'écriture inclusive de l'Administration (artículo 21 de nuestro corpus) publicado el 21 de noviembre de 2011 por la periodista Pech, el mismo día de la publicación del documento oficial. En la tabla siguiente, podemos comprobar que alcanza 561 comentarios internos en la propia $C D L$ y 17 apariciones en Twitter.

\begin{tabular}{|c|c|c|}
\hline Título y tipo $C D L$ & $\begin{array}{c}\text { Commentaires } \\
\text { internes } C D L \\
L^{\prime} \text { Actu des } \\
\text { mots }\end{array}$ & $\begin{array}{c}\text { Feed-back } \\
\text { en Twitter } \\
\text { (commentarios, } \\
\text { publicaciones) }\end{array}$ \\
\hline 1. La langue française a-t-elle un sexe? - Alice Develey & 153 & 14 \\
\hline $\begin{array}{l}\text { 2. Eliane Viennot: "La langue française n'est pas misogy- } \\
\text { ne"-Alice Develey }\end{array}$ & 48 & 13 \\
\hline 3. Et le dictionnaire créa la femme-Alice Develey & 0 & 0 \\
\hline $\begin{array}{l}\text { 4. Bernard Cerquiglini: "L’histoire de la langue française } \\
\text { montre une inégalité homme femme" - Alice Develey }\end{array}$ & 2 & 3 \\
\hline $\begin{array}{l}\text { 5. "Auteure", "autrice”, "écrivaine”: quelle orthographe } \\
\text { employer? - Alice Develey }\end{array}$ & 37 & 7 \\
\hline $\begin{array}{l}\text { 6. Ce que l'année } 2017 \text { a changé dans notre vocabulaire- } \\
\text { Alice Develey }\end{array}$ & 1 & 4 \\
\hline $\begin{array}{l}\text { 7. "Fake news", “bordel”, "féminisme”... Les mots qui ont } \\
\text { marqué } 2017 \text { - Alice Develey }\end{array}$ & 11 & 7 \\
\hline $\begin{array}{l}\text { 8. "Féminisme", mot de l'année aux États-Unis et bientôt } \\
\text { en France? - Alice Develey }\end{array}$ & 13 & 8 \\
\hline
\end{tabular}


Título y tipo $C D L$

Commentaires Feed-back internes $C D L \quad$ en Twitter

L'Actu des (commentarios,

$\begin{array}{lcc} & \text { mots } & \text { publicaciones) }\end{array}$

de la langue" - Alice Develey

10. Alain Rey: "L'écriture inclusive est vouée à l'échec" 234

- Alice Develey

11. Frédéric Vitoux: “C'est faire acte de sagesse que de 103

renoncer à l'écriture inclusive" - Alice Develey

12. Éliane Viennot: "Olympe de Gouges a lancé les prémi- $\quad 296$

ces de l'écriture inclusive" - Alice Develey

13. Danièle Sallenave: "Les femmes doivent être visibles 64

dans la langue française" - Claire Conruyt y Alice Develey

14. L'Académie française prend acte de la féminisation de $\quad 241 \quad 4$

la langue - Claire Conruyt y Alice Develey

15. Auteure, ambassadrice, mannequin... Où en est la fé- $137 \quad 8$

minisation des titres et métiers? - Claire Conruyt y Alice

Develey

16. Dominique Bona: "La féminisation permettra aux fem- 158

mes de sortir d'un malaise linguistique" - Claire Conruyt

17. La Finlande fait don d'un pronom neutre pour encoura- 204

ger l'égalité dans le monde-Lefigaro.fr

18. L'écriture inclusive reste bannie dans les textes du gou- $52 \quad 8$

vernement-Lefigaro.fr

19. L'écriture inclusive, "une langue incompréhensible" 47

pour les non-voyants - Lefigaro.fr

20. L'écriture inclusive est-elle néfaste pour les dyslexi- $137 \quad 7$

ques? - Lefigaro:fr

21. Édouard Philippe bannit l`écriture inclusive de $\mathbf{5 6 1} \quad \mathbf{1 7}$

l>administration - Marie-Estelle Pech

22. Écriture inclusive: Marc Levy pas d'accord avec Ber- 53

nard Pivot - Jean Talabot

23. Du point inclusif au point d'arrêt - Jean Pruvost $\quad 1 \quad 7$

Figura 6: Tabla numérica de las repercusiones internas y externas de los artículos.

\section{Conclusiones}

La primera conclusión a la que llegamos concierne el género de las $C D L$. Nuestras investigaciones demostraron que se trata de un género periodístico que, a pesar de su larga 
tradición, carece de tipología ostensible y de definición en los diccionarios. Asimismo, su amplio y notable campo de estudio deja puertas abiertas a la investigación porque las $C D L$ analizadas en este trabajo han demostrado formar parte de un género libre y evolutivo en función del tiempo, del país y del periódico en el que se enmarcan, tanto que sus características abarcan nuevos parámetros acordes con la nueva era y por consiguiente con las nuevas tecnologías. Gracias a su formato digital, las $C D L$ son dinámicas e interactivas y de fácil acceso a la comunidad hablante quien adquiere cierto protagonismo y puede ser partícipe de los debates planteados.

En base a este parámetro relativo a la participación del lector en los espacios $C D L$ como en sus publicaciones en las redes sociales y considerando el propio tratamiento de la feminización de la lengua ofrecido en los artículos analizados, hemos verificado: a) la capacidad de este género por aportar datos significativos sobre la evolución de los principales aspectos lingüísticos actuales, así como su carácter normativo y su contribución a la codificación y a la estandarización de la lengua, y b) la importancia de las repercusiones en la comunidad lingüística para evaluar el nivel de aceptación de este fenómeno lingüístico en la sociedad. La comunidad lingüística se encuentra por lo tanto en una situación de receptor de ideologías difundidas en las $C D L$ y a la vez de emisor de juicios de valor que repercutan luego en las redes sociales, amplificando así el poder preceptivo y divulgativo de las $C D L$.

Así pues, en un contexto marcado por una revolución terminológica, semántica y hasta sintáctica que supone la feminización del lenguaje, hemos destacado que, para llevarla a cabo, la estrategia lingüística más polémica y que provoca más rechazos es la escritura inclusiva por medio de la doble flexión parcial. En efecto, resulta ser ajena a la realidad social y cambiante que vivimos, porque representa un obstáculo para muchas personas excluyendo así a minorías. De igual modo, la cuestión de la feminización de la lengua en su globalidad recibe la misma crítica por los expertos que no ven en este fenómeno lingüístico ningún beneficio real ni para la lengua, ni para la sociedad. Otros defienden su uso cueste lo que cueste y otros piden moderación y respeto de ciertas reglas y tradición.

Estamos presenciando una época de difícil acuerdo ideológico, en la que la feminización de la lengua no tiene todavía la batalla ganada, Solamente el uso, la paciencia y la moderación serán los aliados de aquella parte de la comunidad lingüística que reclama una reforma para la feminización del lenguaje.

\section{Referencias bibliográficas}

Alpheratz. 2018. "Français inclusif: conceptualisation et analyse linguistique" in $6 e$ Congrès Mondial de Linguistique Française, SHS Web Conf., vol. 46, n⿳013003. < https:// Www.shs-conferences.org/articles/shsconf/pdf/2018/07/shsconf_cmlf2018_13003.pdf> $[02 / 08 / 21]$. 
Anales de Filología Francesa, n. ${ }^{\circ}$ 29, 2021

AURÉLIE FONTANILLE

Revue de linguistique française et d'analyse du discours, tome 11.1: Les défis de l'écriture inclusive" in Lidil, 62 |2020: <http://journals.openedition.org/lidil/8246> [07/08/21].

Becker, Annie \& Bernard Cerquiglini \& Nicole Cholewka et al. 1999. Femme, j'écris ton nom ....: guide d'aide à la féminisation des noms de métiers, titres, grades et fonctions. Paris, La Documentation française: <https://www.vie-publique.fr/sites/default/files/rapport/ pdf/994001174.pdf $>$ [02/08/21].

Boyer, Henri. 2012. 'Idéologie sociolinguistique et politiques linguistiques 'intérieures' de la France" in Cichon, Peter \& Sabine Ehrhart \& Martin Stegu (coord.) Les politiques linguistiques implicites et explicites en domaine francophone, Synergies. Pays germanophones, $n^{\circ}$ 5, Berlin: Gerflint-Avinus, 93-105: <http://www.urbi-beziers.fr/articles/ideologie sociolinguistique_politique_france.pdf $>$ [02/08/21].

Castells, Manuel. 2009. Comunicación y Poder. Madrid, Alianza Editorial: <https:// www.felsemiotica.com/descargas/Castells-Manuel-Comunicaci $\% \mathrm{C} 3 \% \mathrm{~B} 3 \mathrm{n}$-y-poder.pdf> $[09 / 08 / 21]$

Cellard, Jacques. 1983. "Les chroniques de langage” in Bédard, Édith \& Jacques Maurais (coord.) La norme linguistique. Québec/Paris, Conseil de la langue française/Le Robert, 651666: <http://www.cslf.gouv.qc.ca/bibliotheque-virtuelle/publication-html/?tx_iggcpplus_pi4 $\% 5$ bfile $\% 5 d=$ publications/pubf101/f101p5c.html\#xxiv $>$ [02/08/21].

Cerquiglinni, Bernard. 2019. "Bernard Cerquiglini ou l'histoire de la féminisation des mots (rediffusion)" in De vive(s) voix, Radio France Internationale: <http://www.rfi.fr/ emission/20190307-bernard-cerquiglini-histoire-feminisation-mots $>$ [01/05/21].

Grijelmo, Álex. 2007. "Intervención en Cartagena" in Donde dice... Revista de la Fundación del Español Urgente, $\mathrm{n}^{\circ}$ 8, 1-3: < https://docplayer.es/6510705-Boletin-de-la-fundacion-delespanol-urgente-1-m-lengua-de-un-pueblo-o-nacion-o-comun-a-varios.html $>$ [02/08/21] .

Guerrero Salazar, Susana. 2020. "El debate social en torno al lenguaje no sexista en la lengua española" in IgualdadES, 2, 201-221. <https://doi.org/10.18042/cepc/IgdES.2.07> [08/08/21].

Gutiérrez-Rubí, Antoni. 2008. "Lecciones de la campaña de Obama”, in Revista Fundació Rafael Campalans, 16, 75-82: < https://fcampalans.cat/uploads/publicacions/pdf/frc17_gutierrezrubi.pdf> [10/08/21].

Hugonnier, Claire. 2019. "Danièle Manesse et Gilles Siouffi (dir.), Le féminin et le masculin dans la langue: 1'écriture inclusive en questions" in Lidil n ${ }^{\circ} 62 \mid 2020<$ http://journals. openedition.org/lidil/8172> [07/08/20].

LóPez PAN, Fernando. 1995. 70 columnistas de la prensa española. Pamplona, EUNSA

LuQue DurÁn, Juan de Dios. 2004. “Aspectos universales y particulares de las lenguas del mundo" in Estudios de Lingüística del Español, 21: <http://elies.rediris.es/elies21/CAPITULO11.pdf> [02/08/21].

Mancera Rueda, Ana, \& Ana Pano Alemán. 2014. "Nuevas dinámicas discursivas en la comunicación política en Twitter" in Círculo De Lingüística Aplicada a La Comunicación, 56, 53-80. <https://doi.org/10.5209/rev_CLAC.2013.v56.43867> [10/08/21].

Mathieu, Marie Joe \& Jean Marie PIERREL. 2009. "La féminisation dans la langue” in 
Guittienne Lucile \& Marlène Proust (coord.) Homme-femme: de quel sexe êtes-vous? in PUN, 111-122. halshs-00397463f. <https://halshs.archives-ouvertes.fr/halshs-00397463/file/ La_Feminisation_dans_la_langue.pdf $>$ [02/08/21].

Marimón Llorca, Carmen. 2019. El columnismo lingüístico en España desde 1940. Análisis multidimensional y caracterización genérica. Madrid, Arco Libros.

Morán Torres, Esteban. 1988. Géneros del periodismo de opinión. Crítica, comentario, columna, editorial. Pamplona, EUNSA.

Paveau, Marie Anne. 2013. "Genre de discours et technologie discursive. Tweet, twittécriture et twittérature" in Théories et pratiques des genres $\mathrm{n}^{\circ} 157-158,7-30:<\mathrm{https}$ ://journals. openedition.org/pratiques/3533> [02/08/21].

QuemadA, Bernard. 1970-1972. Bibliographie des chroniques de langage publiées dans la presse française, vol. 1 (1950-1965), Paris, Didier ("Centre d'études du français moderne et contemporain. Etudes et documents, 1"), 1970, xxx-419; vol. 2 (1966-1970), Paris, Didier ("Publications du Centre d'études du français moderne et contemporain, 5"), 1972, xxxii-279.

ReMysen, Wim. 2009. Description et évaluation de l'usage canadien dans les chroniques de langage: contribution à l'étude de l'imaginaire linguistique des chroniqueurs canadiensfrançais. Thèse de doctorat, Québec, Université de Laval.

Sánchez Manzanares, Carmen. 2019. "La unidad del idioma y otras ideologías lingüísticas en las columnas sobre la lengua de Álex Grijelmo" in Marimón Llorca Carmen \& Santamaría Pérez Isabel (coord.) Ideologías sobre la lengua y medios de comunicación escritos: El caso del español. Berlin, Peter Lang, 217-234.

SANTAMARÍA PÉRez, Isabel. 2019. "Baia baia, ¿Irreverencia o progreso?: Nuevas autoridades en las CSL del siglo XXI" in Marimón Llorca Carmen (ed.) El columnismo lingüístico en España desde 1940. Análisis multidimensional y caracterización genérica. Madrid, Arco Libros, 231-257.

\section{Anexo: Títulos, autores y referencias de cada artículo que forma el corpus} [16.05.2021].

1 La langue française a-t-elle un sexe? - Alice Develey

$<$ https://www.lefigaro.fr/langue-francaise/actu-des-mots/2019/02/05/37002-

20190205ARTFIG00015-la-langue-francaise-a-t-elle-un-sexe.php>

2 Eliane Viennot: "La langue française n'est pas misogyne" - Alice Develey

$<$ https://www.lefigaro.fr/langue-francaise/actu-des-mots/2018/11/19/37002-

20181119ARTFIG00027-eliane-viennot-la-langue-francaise-n-est-pas-misogyne. php> 
3 Et le dictionnaire créa la femme - Alice Develey $<$ https://www.lefigaro.fr/langue-francaise/actu-des-mots/2018/03/07/3700220180307ARTFIG00008-et-le-dictionnaire-crea-la-femme.php>

4 Bernard Cerquiglini: "L'histoire de la langue française montre une inégalité homme femme" - Alice Develey

$<$ https://www.lefigaro.fr/langue-francaise/actu-des-mots/2018/10/10/3700220181010ARTFIG00046-bernard-cerquiglini-1-histoire-de-la-langue-francaisemontre-une-inegalite-homme-femme.php>

5 “Auteure”, “autrice”, "écrivaine”: quelle orthographe employer? - Alice Develey $<$ https://www.lefigaro.fr/langue-francaise/actu-des-mots/2018/03/07/3700220180307ARTFIG00093-auteure-autrice-ecrivaine-quelle-orthographe-employer. php>

6 Ce que l'année 2017 a changé dans notre vocabulaire - Alice Develey $<$ https://www.lefigaro.fr/langue-francaise/actu-des-mots/2017/12/30/3700220171230ARTFIG00003-ce-que-1-annee-2017-a-change-dans-notre-vocabulaire. php>

7 "Fake news", “bordel”, “féminisme”... Les mots qui ont marqué 2017 - Alice Develey

$<$ https://www.lefigaro.fr/langue-francaise/actu-des-mots/2017/12/21/3700220171221ARTFIG00006-fake-news-bordel-feminisme-les-mots-qui-ont-marque-2017.php>

8 "Féminisme", mot de l'année aux États-Unis et bientôt en France? - Alice Develey $<$ https://www.lefigaro.fr/langue-francaise/actu-des-mots/2017/12/14/3700220171214ARTFIG00064-feminisme-mot-de-l-annee-aux-etats-unis-et-bientot-enfrance.php>

9 Loïc Depecker: “On ne peut pas préjuger de l'évolution de la langue” — Alice Develey

$<$ https://www.lefigaro.fr/langue-francaise/actu-des-mots/2017/11/30/3700220171130ARTFIG00005-loic-depecker-on-ne-peut-pas-prejuger-de-l-evolution-dela-langue.php>

10 Alain Rey: "L'écriture inclusive est vouée à l'échec" - Alice Develey $<$ https://www.lefigaro.fr/langue-francaise/actu-des-mots/2017/11/23/3700220171123ARTFIG00015-alain-rey-1-ecriture-inclusive-est-vouee-a-1-echec.php> 
11 Frédéric Vitoux: "C'est faire acte de sagesse que de renoncer à l'écriture inclusive" - Alice Develey

$<$ https://www.lefigaro.fr/langue-francaise/actu-des-mots/2017/11/22/3700220171122ARTFIG00006-frederic-vitoux-c-est-faire-acte-de-sagesse-que-de-renoncer-a-l-ecriture-inclusive.php>

12 Éliane Viennot: “Olympe de Gouges a lancé les prémices de l'écriture inclusive”Alice Develey

$<$ https://www.lefigaro.fr/langue-francaise/actu-des-mots/2017/11/05/3700220171105ARTFIG00005-eliane-viennot-olympe-de-gouges-a-lance-les-premicesde-1-ecriture-inclusive.php>

13 Danièle Sallenave: "Les femmes doivent être visibles dans la langue française" Claire Conruyt y Alice Develey

$<$ https://www.lefigaro.fr/langue-francaise/actu-des-mots/2019/03/08/3700220190308ARTFIG00027-daniele-sallenave-les-femmes-doivent-etre-visibles-dansla-langue-francaise.php>

14 L'Académie française prend acte de la féminisation de la langue - Claire Conruyt y Alice Develey

$<$ https://www.lefigaro.fr/langue-francaise/actu-des-mots/2019/02/28/3700220190228ARTFIG00244-1-academie-francaise-prend-acte-de-la-feminisation-dela-langue.php>

15 Auteure, ambassadrice, mannequin... Où en est la féminisation des titres et métiers? - Claire Conruyt y Alice Develey

$<$ https://www.lefigaro.fr/langue-francaise/actu-des-mots/2019/02/27/3700220190227ARTFIG00209-auteure-ambassadrice-mannequin-o-en-est-la-feminisation-des-titres-et-metiers.php>

16 Dominique Bona: "La féminisation permettra aux femmes de sortir d'un malaise linguistique”- Claire Conruyt

$<$ https://www.lefigaro.fr/langue-francaise/actu-des-mots/2019/02/20/3700220190220ARTFIG00028-dominique-bona-la-feminisation-permettra-aux-femmesde-sortir-d-un-malaise-linguistique.php>

17 La Finlande fait don d'un pronom neutre pour encourager l'égalité dans le monde - Lefigaro.fr

$<$ https://www.lefigaro.fr/langue-francaise/actu-des-mots/la-finlande-fait-don-d-unpronom-neutre-pour-encourager-l-egalite-dans-le-monde-20190603> 
18 L'écriture inclusive reste bannie dans les textes du gouvernement - Lefigaro.fr $<$ https://www.lefigaro.fr/langue-francaise/actu-des-mots/2019/02/28/3700220190228ARTFIG00247-1-ecriture-inclusive-rejetee-par-le-conseil-d-etat.php>

19 L'écriture inclusive, "une langue incompréhensible” pour les non-voyants - Lefigaro.fr

$<$ https://www.lefigaro.fr/langue-francaise/actu-des-mots/2017/11/21/3700220171121ARTFIG00088-1-ecriture-inclusive-une-langue-incomprehensible-pourles-non-voyants.php>

20 L'écriture inclusive est-elle néfaste pour les dyslexiques? - Lefigaro.fr $<$ https://www.lefigaro.fr/langue-francaise/actu-des-mots/2017/10/30/3700220171030ARTFIG00092-1-ecriture-inclusive-est-elle-nefaste-pour-les-dyslexiques. php>

21 Édouard Philippe bannit l>écriture inclusive de l>administration - Marie-Estelle Pech <https://www.lefigaro.fr/actualite-france/2017/11/21/01016-20171121ARTFIG00179-edouard-philippe-bannit-l-ecriture-inclusive-de-l-administration.php>

22 Écriture inclusive: Marc Levy pas d'accord avec Bernard Pivot - Jean Talabot $<$ https://www.lefigaro.fr/langue-francaise/actu-des-mots/2017/11/12/3700220171112ARTFIG00111-ecriture-inclusive-marc-levy-pas-d-accord-avec-bernardpivot.php>

23 Du point inclusif au point d'arrêt - Jean Pruvost $<$ https://www.lefigaro.fr/langue-francaise/actu-des-mots/2017/11/24/3700220171124ARTFIG00106-du-point-inclusif-au-point-d-arret.php> 
